\title{
Production of Large Diameter ECR Plasma
}

\author{
Y. Kawai and Y. Ueda
}

Interdisciplinary Graduate School of Engineering Sciences, Kyushu University, Kasuga, Fukuoka 816, Japan

\begin{abstract}
The production of a large diameter ECR plasma with microwaves of frequency $2.45 \mathrm{GHz}$ is reviewed, including our experimental results with a multi-slot antenna and a $\mathrm{TE}_{01}$ mode microwave converter. It is pointed out that the electromagnetic waves play an important role in plasma uniformity as well as plasma production. Furthermore, it is found that the $\mathrm{X}$ wave contributes to plasma uniformity for electron density of (1-2) $\times 10^{17} \mathrm{~m}^{-3}$ which almost corresponds to the $\mathrm{L}$ cutoff.
\end{abstract}

\section{INTRODUCTION}

Recently the development of a large diameter plasma source has become one of the most important subjects in plasma processing such as etching and CVD. Above all, there has been great interest in an electron cyclotron resonance (ECR) plasma [1-3] because of the high density plasma at low pressure $(<5 \mathrm{mT}$ orr) and quite low ion energy (tens of $\mathrm{eV}$ ) at the wafer, compared with the RF plasma. Usually the principal mode in waveguides, $\mathrm{TE}_{10}$ or $\mathrm{TE}_{11}$ mode, is adopted to produce an ECR plasma [1-3]. In this case, the wavelength of microwaves in vacuum is about $120 \mathrm{~mm}$ at $2.45 \mathrm{GHz}$ so that it is hard to produce an ECR plasma whose diameter is more than $120 \mathrm{~mm}$ without any ideas. Now, a large diameter plasma with the diameter more than $200 \mathrm{~mm}$ has been required for etching and CVD from industry. Thus, the production of an ECR plasma whose diameter is more than $200 \mathrm{~mm}$ has become interesting topic in plasma processing.

Many attempts [4-11] to produce an ECR plasma of $200 \mathrm{~mm}$ in diameter were made and interesting results were reported. We also developed two types of ECR plasma sources using a multi-slot antenna [56] and a circular $\mathrm{TE}_{01}$ mode microwave [7-8]. A multi-slot antenna has an advantage that the diameter of the antenna can be chosen independently of the microwave frequency so that the production of a large diameter ECR plasma is expected. In fact, a large diameter uniform plasma $200 \mathrm{~mm}$ in diameter was produced with a multi-slot antenna $280 \mathrm{~mm}$ in diameter [6,9]. Furthermore, it was found [9] by measuring wave patterns that both the whistler wave ( $R$ wave) and the $L$ wave(Left-handed wave) [12] exist in the plasma.

A high plasma density such as $10^{18} \mathrm{~m}^{-3}$ is necessary for higher speed etching or deposition. In order to realize a uniform and dense ECR plasma, we developed a new type of ECR plasma source [7] using a circular $T E_{01}$ microwave mode which is converted from the principal rectangular mode $T E_{10}$. The ion saturation current density of $360 \mathrm{~A} / \mathrm{m}^{2}$ was achieved for the input microwave power of $3 \mathrm{~kW}$ at nitrogen pressure of $5 \times 10^{-4}$ Torr. The uniformity of the ion saturation current density was within $\pm 3 \%$ over 200 $\mathrm{mm}$ in diameter. It was also confirmed that when the electron density is higher than $10^{17} \mathrm{~m}^{-3}$, the whistler wave ( $R$ wave) propagates, while when the electron density is lower than $10^{17} \mathrm{~m}^{-3}$, both the $R$ wave and $L$ wave propagate [8].

Above mentioned results suggest that the electromagnetic waves such as the $\mathrm{R}$ wave and $\mathrm{L}$ wave play an Article published online by EDP Sciences and available at http://dx.doi.org/10.1051/jp4:1997419 
important role in plasma production. Aydil et al [13] investigated how the radial profile of the ion saturation current depends on input microwave powers and discussed the relationship between the radial profile and the $L$ wave. However, it is still not clear what the dominant mechanisms are in ECR plasma uniformity. In order to clarify the physical mechanism of ECR plasma uniformity, we have performed the experiments on the propagation of the electromagnetic waves in the radial direction as well as in the axial direction, where an ECR plasma was produced with a conventional $\mathrm{TE}_{11}$ mode. Recently, it was found [14] that the extraordinary wave ( $X$ wave) mainly contributes to plasma uniformity around the electron density of $10^{17} \mathrm{~m}^{-3}$, which corresponds to the cutoff density of the $X$ wave ( $L$ cutoff) [12]. This result will be useful for producing a $300 \mathrm{~mm}$ diameter ECR plasma which has been required as a next generation plasma source from industry.

In this paper, we describe some attempts of producing a large diameter ECR plasma, including our experiments, and point out that the $\mathrm{X}$ wave as well as the $\mathrm{R}$ wave contributes to plasma uniformity.

\section{ATTEMPTS OF PRODUCING A LARGE DIAMETER ECR PLASMA}

\subsection{Typical results on the production of a $200 \mathrm{~mm}$ diameter ECR plasma}

Matsuo et al [3] developed an ECR plasma source for plasma processing using a divergent magnetic field configuration. Microwave powers (frequency $2.45 \mathrm{GHz}, \mathrm{TE}_{11}$ mode) were introduced into the plasma chamber through a waveguide and a quartz window. Then, a highly activated plasma was produced at low gas pressures in the range from $10^{-5}$ to $10^{-3}$ Torr. The plasma parameters were : $n_{e}=10^{16}-10^{18} \mathrm{~m}^{-3}, T_{e}=5-10$ $\mathrm{eV}$ and plasma potential $\mathrm{V}_{\mathrm{p}}=15-20 \mathrm{~V}$. Now this ECR plasma system has been widely used for etching and CVD. However, this conventional ECR plasma source does not provide a uniform plasma because a divergent magnetic field distribution is used as a magnetic field configuration.

Samukawa et al. [4] succeeded in the production of a large diameter ECR plasma of $200 \mathrm{~mm}$ in diameter by optimizing both the size of the discharge chamber and the gradient of the magnetic field. In this case, note that the 875 gauss equimagnetic field plane was located on the substrate holder, that is, uniform magnetic fields were adopted instead of divergent magnetic fields. They obtained the ion saturation current density of $160 \mathrm{~A} / \mathrm{m}^{2}$ at nitrogen gas pressure of $5 \times 10^{-4}$ Torr, where the uniformity of $\pm 5 \%$ was achieved. Furthermore, they carried out the etching using their system and obtained a lot of interesting results [4].

There were many attempts [5-11] to produce an ECR plasma whose diameter is larger than 6 inches with other methods. Plasma sources employing multi-pole magnetic fields are compact and less expensive. Furthermore, magnetic field coils to confine plasmas are not necessary. Sato et al [10-11] tried to produce a large diameter plasma with a plane multi-slot antenna and obtained the uniformity within $\pm 3 \%$ for a diameter of $200 \mathrm{~mm}$. A microwave of $2.45 \mathrm{GHz}$ traveling through a rectangular waveguide was converted into a coaxial mode. The plane antenna consisted of a back-plate with permanent magnets behind and a slotted plate connected to the inner electrode of the coaxial waveguide. The antenna surface was covered with a glass plate of $1 \mathrm{~mm}$ thickness to prevent direct contact with plasma particles. The ECR condition was satisfied in a limited region within $10 \mathrm{~mm}$ from the magnet surface. The density and temperature of electrons were about $2 \times 10^{16} \mathrm{~m}^{-3}$ and $3 \mathrm{eV}$, respectively, at the microwave power of $50 \mathrm{~W}$ under the argon pressure of $10^{-4}$ Torr. The plasma density was almost proportional to the microwave power. They succeeded in producing a uniform ECR plasma of 400-450 mm (16-18 inches) in diameter with a plane slot antenna. However, the physical mechanism of plasma uniformity has not been clarified, so far.

\subsection{Production of a large diameter ECR plasma with a multi-slot antenna}

We have succeeded in producing a uniform and large diameter ECR plasma using a large diameter multislot antenna (MSA). The MSA has an advantage that the plasma diameter does not depend on the frequency of the incident microwave [5-6]. So far, the MSA has been regarded to be an assembly of a lot 
of thin-slot antennas distributed uniformly on the surface of a cylinder [5]. A uniform plasma (8 inches, \pm $5 \%$ ) was realized [9] by the MSA under the optimized conditions of microwave power, pressure and magnetic field configuration. Especially, the uniformity of the radial profile of the ion saturation current density depended greatly on magnetic field configurations. It was also found that the ion saturation current density axially increased after the resonance point under the certain magnetic field profiles. Usually, an ECR plasma has been produced by two methods: resonant acceleration of electrons by microwave electric fields, and resonant interaction between the whistler wave and electrons. The mechanism of the production of an ECR plasma using a multi-slot antenna has been considered as the former. The above mentioned results suggest that there may exist normal modes relating to the plasma production. Thus, we examined the effect of the magnetic field configuration on the uniformity of the ion saturation current density in order to clarify the mechanism of the ECR plasma production using the MSA. Furthermore, it was examined by measuring the dispersion relation of electromagnetic waves whether normal modes in the plasma exist or not.

A schematic diagram of the experimental apparatus is shown in Fig. 1(a). The vacuum chamber is made of stainless steel with $290 \mathrm{~mm}$ in inner diameter and $1200 \mathrm{~mm}$ in length. An ECR plasma was produced with a multi slot antenna which is made of stainless steel of $280 \mathrm{~mm}$ in diameter, as seen in Fig. 1(b). The length and width of slots are $70 \mathrm{~mm}$ and $2 \mathrm{~mm}$, respectively. The magnetic coil assembly consisted of six coils; some coils to make uniform magnetic fields, while, the other to form the magnetic mirror and control the current for magnetic mirror. The gas used was $\mathrm{He}$, and the typical pressure was $2 \times 10^{-4}$ Torr. In this experiment, the substrate which is at floating was placed at $800 \mathrm{~mm}$ from the multi-slot antenna $(\mathrm{Z}=1000$ $\mathrm{mm}$ ). In discussing a large diameter plasma, it is important whether or not a substrate is placed in the chamber, because the plasma is disturbed by the substrate. Here we examined the uniformity of plasma in the presence of a substrate of 8 inches in diameter. The ion saturation current density, $I_{i s}$, in the absence of the substrate were two times larger than in the presence of it. Plasma parameters were measured with a cylindrical Langmuir probe in front of the substrate. Electromagnetic waves in the plasma were measured with a loop antenna movable along $\mathrm{z}$ axis and the wave patterns were obtained by the interferometer method [15] as usual.

It was achieved under the gas pressure $2 \times 10^{-4}$ Torr, $2 \times 10^{-3}$ Torr and microwave power $470 \mathrm{~W}, 1000 \mathrm{~W}$ that the radial uniformity of the ion saturation current density is within $5 \%$ over $200 \mathrm{~mm}$ in diameter, as seen in Fig.2(a). The electron density and the electron temperature of the uniform plasma in front of the substrate were $7 \times 10^{16} / \mathrm{m}^{3}$ and $6 \mathrm{eV}$, respectively. The radial profile of the floating potential, $\mathrm{V}_{\mathrm{f}}$, in the case of $470 \mathrm{~W}$ where the plasma is uniform is shown in Fig.2(b), which indicates that the profile of $V_{\mathrm{f}}$ is uniform, $\pm 0.75 \mathrm{~V}$ over $200 \mathrm{~mm}$. The value of $V_{f}$ are slightly positive although $V_{f}$ in the case where the radial profiles are not uniform were in the range from $-5 \mathrm{~V} \sim-20 \mathrm{~V}$, which means that there are little high energy electrons under the condition of the uniform plasma.

It has been pointed out $[13,16]$ that the whistler wave (or $R$ wave) plays an important role in the production of a large diameter ECR plasma. As already mentioned, it seems that a multi-slot antenna can excite normal modes in the plasma. Thus, electromagnetic waves in the plasma were measured [9] with a small loop antenna in order to clarify the dependence of the radial profiles of $I_{\text {is }}$ on the magnetic field configurations from the point of view of plasma production. Figure 3(a) is a typical interferometric wave pattern of $B_{x}$ in the uniform plasma. As seen in Fig.3(b), the wave pattern was decomposed graphically into two traces assuming that it consisted of two waves with different wave numbers. Figures $4(\mathrm{a})$ and $4(\mathrm{~b})$ show the dispersion relations before the ECR point and after the ECR point where the solid line is the theoretical dispersion curve. Here, the local wave numbers were estimated from the wave patterns in the regions between the MSA and ECR point and after ECR point. Figure 4 shows that both the R-wave and the L-wave propagate in the region between the MSA and ECR point, and the only L-wave propagates after the ECR point, which is the same results as those obtained with a conventional ECR plasma source [15]. 
Thus, it is concluded from above results that the MSA is regarded a sort of microwave antennas with higher order modes.

\subsection{Production of a high density-large diameter ECR plasma with $\mathrm{TE}_{01}$ mode}

It is a little hard to produce a plasma whose density is higher than $10^{17} \mathrm{~m}^{-3}$ with a multi-slot antenna. On the other hand, higher density plasma will be required from industry, in particular for plasma CVD or sputtering. We have developed a new ECR plasma source using a circular $\mathrm{TE}_{01}$ mode and succeeded in the production of a uniform high density ECR plasma. A microwave source of $2.45 \mathrm{GHz}$ frequency was used to generate an ECR plasma. Microwaves generated by the magnetron propagate into an ECR plasma through the waveguides. There are many waveguide modes of microwaves, such as TE mode and TM mode. In the case of a conventional ECR plasma source, microwaves are introduced into cylindrical chambers as the circular $\mathrm{TE}_{11}$ mode converted from the rectangular $\mathrm{TE}_{10}$ mode. In the case of the $\mathrm{TE}_{11}$ mode, the electric field has a strong peak at the center of the waveguide, which causes the peaking of the plasma density at the central region. On the other hand, in the case of the $\mathrm{TE}_{01}$ mode, the electric field near the wall is stronger than that at the center of the waveguide. This strong electric field near the wall may compensate the density decrease near the chamber wall. Furthermore, as for the $\mathrm{TE}_{01}$ mode, the electric field distribution along the circumferential $(\theta)$ direction is uniform at the same radial position. Conversely, the $\mathrm{TE}_{11}$ mode has a different electric field strength for different $\theta$ values. Consequently the $\mathrm{TE}_{01}$ mode will be more convenient in generating a uniform ECR plasma.

Figure 5 shows the experimental apparatus. The microwave power of $2.45 \mathrm{GHz}$ could be varied up to 5 $\mathrm{kW}$. The rectangular $\mathrm{TE}_{10}$ mode was converted to the circular $\mathrm{TE}_{01}$ mode and introduced into the ECR plasma source. The diameter and length of the ECR plasma source are $300 \mathrm{~mm}$ and $620 \mathrm{~mm}$, respectively. The distance between the magnetic coils and the substrate is variable along the $z$ axis. The magnetic field profile was controlled by changing the coil current and the coil locations. The base pressure was kept less than $1 \times 10^{-7}$ Torr. The ion saturation current density and the plasma potential were measured by a plane Langmuir probe $1 \mathrm{~mm}$ in diameter.

Actually we measured the electric field distribution of the microwave emitted from both the $\mathrm{TE}_{01}$ and $\mathrm{TE}_{11}$ mode converter. Figure 6 is typical profiles of the measured electric field distributions. Because of the interference by the probing microwave antenna, the distributions are not always symmetric. It is obvious that the electric field intensity from the $\mathrm{TE}_{01}$ mode converter has a couple of peaks around $R= \pm 65 \mathrm{~mm}$ and is almost zero at $\mathrm{R}=0 \mathrm{~mm}$. As predicted, the electric field of the $T E_{11}$ mode converter peaks at $R=0$ $\mathrm{mm}$.

Figure 7 shows the dependence of the ion saturation current density on the input microwave power. The nitrogen gas pressure is $5 \times 10^{-4}$ Torr and the ion saturation current density is measured at $Z=350 \mathrm{~mm}$ and $\mathrm{R}=0 \mathrm{~mm}$. As seen in Fig.7, the ion saturation current density increases nearly in proportion to the input microwave power. In order to examine the uniformity of the ECR plasma, the radial distribution of the ion saturation current density was measured with a movable Langmuir probe. The result is shown in Fig. 8 for different microwave modes. In this case, the input microwave power was $3 \mathrm{~kW}$ and the nitrogen gas pressure was $5 \times 10^{-4}$ Torr. In Fig.8(a), note that the ion saturation current density is uniform within \pm $3 \%$ over $200 \mathrm{~mm}$ in diameter and $360 \mathrm{~A} / \mathrm{m}^{2}$ is achieved with the $\mathrm{TE}_{-3}$ mode. The measured electron temperature $\left(\mathrm{T}_{\mathrm{e}}\right)$ and the plasma density $\left(\mathrm{N}_{\mathrm{e}}\right)$ were $6 \mathrm{eV}$ and $6 \times 10^{17} \mathrm{~m}^{-3}$, respectively.

On the other hand, the ion saturation current density distribution with the $\mathrm{TE}_{11}$ mode is less uniform. Note here that all external parameters were the same except for the microwave mode. Thus, it is obvious that the $\mathrm{TE}_{01}$ mode is better than the $\mathrm{TE}_{11}$ mode from the standpoint of obtaining a radially uniform plasma.

To clarify the production mechanism of a large diameter uniform ECR plasma generated by a circular $\mathrm{TE}_{01}$ mode microwave experimentally, wave propagation characteristics in the plasmas were measured, as 
in the case of a multi-slot antenna. It was found [8] that a microwave introduced into the chamber is transformed mainly into the electron cyclotron wave (R-wave) and then absorbed around the resonant point.

In conclusion, it was found that an ECR plasma source with a circular $\mathrm{TE}_{01}$ mode is very convenient for achieving a high and uniform ion current density. The ion saturation current density was uniform within \pm $3 \%$ over $200 \mathrm{~mm}$ in diameter and $360 \mathrm{~A} / \mathrm{m}^{2}$ was achieved.

\section{MECHANISM OF PLASMA UNIFORMITY}

As described in Sec.2, an ECR plasma of $200 \mathrm{~mm}$ in diameter came to be realized experimentally with a multi-slot antenna and a $\mathrm{TE}_{01}$ mode converter. However, the conditions where such a uniform ECR plasma is produced strongly depended on the external conditions such as magnetic field distribution, pressure, input microwave power and so on. In addition, it seems that the electromagnetic waves play an important role in ECR plasma uniformity. Stevens and Cecchi [15] pointed out that the $R$ wave contributes to plasma uniformity by calculating the ray trace of the $R$ wave. Aydil et al [13] and Carl et al [18] found a uniform profile of the ion saturation current and tried to explain it by the $\mathrm{L}$ wave although electromagnetic waves were not measured. Thus, we have performed the experiments on electromagnetic wave measurements in order to clarify the physical mechanism of plasma uniformity. It was found that the extraordinary wave ( $\mathrm{X}$ wave) radially propagates and contributes to plasma uniformity.

Figure 9 shows the experimental apparatus which is the same apparatus as that of Sec.2.2 except for the introduction of the microwaves. In this case, the $\mathrm{TE}_{11}$ mode was adopted in order to compare with the experimental results $[13,15,18]$ reported so far . The microwave power could be varied up to $5 \mathrm{~kW}$. The used gas was $\mathrm{N}_{2}$ and the pressure was ranged from 0.5 to $5 \mathrm{~m}$ Torr. An electron cyclotron resonance point was set at $Z=230 \mathrm{~mm}$. The plasma parameters were measured with a $1 \mathrm{~mm}$-diam Langmuir probe. The eleetric fields were picked up with a movable loop antenna connected to a crystal diode or a mixer and the wave patterns were observed by the interferometer method $[8-9,15]$ to obtain the dispersion relation of electromagnetic waves. In this experiment, a phase shifter was used to measure whether waves propagate or not.

The radial profile of the ion saturation current density $I_{i s}$ was examined as a function of input microwave power. It was found that as the input microwave power was increased, the radial profile of lis became initially convex, wavy, concave, uniform and finally convex, which is the same tendency as observed so far $[13,18]$. Whenever the radial profile of Iis was uniform around the center, it had a peak near the chamber, as shown below. In order to clarify such behavior, we measured the radial propagation of electromagnetic waves using a radially movable loop antenna which was installed at $Z=450 \mathrm{~mm}$. Figure 10 and Fig. 11 show the interferometric wave patterns for different input microwave powers $P_{\text {in, }}$, where the wave patterns of electromagnetic waves were taken by changing the phase of the reference signal with the phase shifter. These figures indicate that there are radially propagating waves. Note that as seen in Fig.11(a), the wavelength of the waves is very short near the chamber wall,while it is long around the center. Furthermore, Fig.11(b) shows that the output of the crystal diode which is proportional to the electric field intensity of waves peaks near the chamber wall. On the other hand, Fig. 12 shows that the radial profile of $I_{i s}$ peaks near the chamber wall. Thus, it turns out that the peak of $I_{i s}$ corresponds to that of the electric field. When the input microwave power was increased further and the electron density exceeded about $2 \times 10^{17} \mathrm{~m}^{-3}$, the radially propagating waves disappeared. In this case, the $\mathrm{L}$ wave propagating along the axial direction was not excited and only the $\mathrm{R}$ wave propagated to the electron cyclotron resonance point.

In order to identify what kind of electromagnetic waves were excited, the dispersion relation was plotted for different electron densities. Here the wavelength was estimated from the wave patterns of Fig. 10 and Fig.11. The obtained dispersion relation agrees with the theoretical one of the $\mathrm{X}$ wave, as seen in Fig. 13 where $\omega_{\mathrm{se}}$ and $\mathrm{k}_{\mathrm{x}}$ are the electron cyclotron frequency and wavenumber of the $\mathrm{X}$ wave. According to the 
linear theory[12], when the wavelength of the $\mathrm{X}$ wave becomes short, upper hybrid resonance takes place. Thus, a peak of the ion saturation current density near the chamber wall is considered to be caused by upper hybrid resonance. The observed cutoff density, $2 \times 10^{17} \mathrm{~m}^{-3}$, at which the $\mathrm{X}$ wave disappeared corresponds to the $\mathrm{L}$ cutoff [12]. In conclusion, the $\mathrm{X}$ wave contributes to ECR plasma uniformity around the cutoff density of the $\mathrm{X}$ wave and the $\mathrm{L}$ wave does not play a dominant role in plasma uniformity.

\section{CONCLUSION}

We reviewed the production of an ECR plasma whose diameter is $200 \mathrm{~mm}$ with microwaves of frequency of $2.45 \mathrm{GHz}$ and pointed out that the $\mathrm{R}$ wave plays an important role in the plasma production. Furthermore, it was found the $\mathrm{X}$ wave contributes to ECR plasma uniformity for the electron density around the L cutoff, (1-2) $\times 10^{17} \mathrm{~m}^{-3}$, which will be useful for designing larger diameter ECR plasma sources. Recently, it seems that Watanabe et al at Hitachi [19] succeeded in producing a $300 \mathrm{~mm}$ diameter ECR plasma with $\mathrm{TE}_{01}$ mode [7]. An ECR plasma with the electron density higher than the L cutoff will be necessary for CVD to increase the deposition rate, which is one of future problems concerning ECR plasma sources.

\section{References}

[1] SAKUDO N., TOKIGUCHI K., KOIKE H. and KANOMATA I, Rev. Sci. Instrum., 48 (1977) 762766.

[2] SUZUKU K., OKUDAIRA S., SAKUDO N. and KANOMATA I., Jpn. J. Appl. Phys., 16 (1977) 1979-1984.

[3] MATSUO S. and KIUCHI M., Jpn. J. Appl. Phys., 22 (1983) L210-L212.

[4] SAMUKAWA S. , J. Vac. Sci. Technol., B8 (1990) 1192-1198.

[5] YONESU A., TAKEUCHI Y., KOMORI A. and KAWAI Y., Jpn. J. Appl. Phys., 27 (1988) L1746L1749.

[6] UEDA Y., TANAKA M., SHINOHARA S. and KAWAI Y., Surface and Coating Technology, 74-75 (1995) 503-507.

[7] HIDAKA R., YAMAGUCHI T., HIROTSU N., OHSHIMA T., KOGA R., TANAKA M. and Kawai Y.; Jpn. J. Appl. Phys., 32 (1993) 174-178.

[8] HIROTSU N., YAMAGUCHI T., HIDAKA R., TANAKA M. and KAWAI Y., Jpn. J. Appl. Phys., 33 (1994) 2712-2717.

[9] UEDA Y., TANAKA M., SHINIHARA S. and KAWAI Y., Rev. Sci. Instrum. 66 (1995) 5423-5427.

[10] IIZUKA S. and SATO N., , Jpn. J. Appl. Phys., 33 (1994) 4221-422 .

[11] ISHIDA T.,NAKAGAWA Y., ONO T., IIZUKA S. and SATO N. , Jpn.J. Appl.Phys., 33 (1994) 4236-4238.

[12] CHEN F.F. Introduction To Plasma Physics (Plenum Press, New York and London, 1974), Chap.4.

[13] AYDIL E.S., GREGUS J.A. and GOTTSCHO R.A., Rev. Sci. Instrum., 64 (1993) 3572-3584.

[14] UEDA Y. and KAWAI Y., to be published in Proc. of ICPIG (Toulouse, July, 1997).

[15] STEVENS J.E.and CECCHI J.L. Jpn. J. Appl. Phys., 32 (1993) 3007-3012.

[16] SAMUKAWA S., J. Vac. Sci. Technol., A11 (1993) 2572-2576.

[17] UEDA Y. and KAWAI Y., to be published in Appl. Phys. Lett. (1997). 
[18] CARL D.A., WILLIAMSON M.C., LIEBERMAN M.A. and LICHTENBERG A.J., J. Vac. Sci. Technol., B9 (1991) 339-347.

[19] WATANABE S. et al., to be published in Jpn. J. Appl. Phys. (1997).

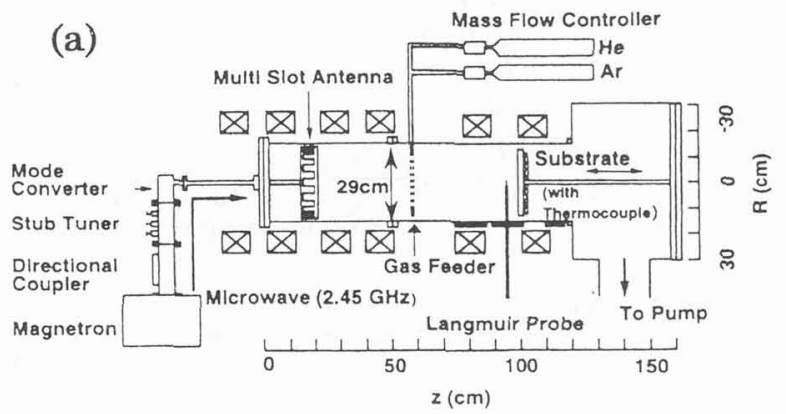

(b)

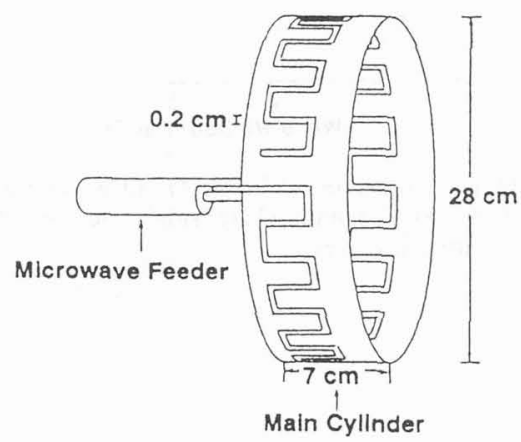

Fig. 1. Schematic diagram of (a) the experimental apparatus and (b) the multi-slot antenna.
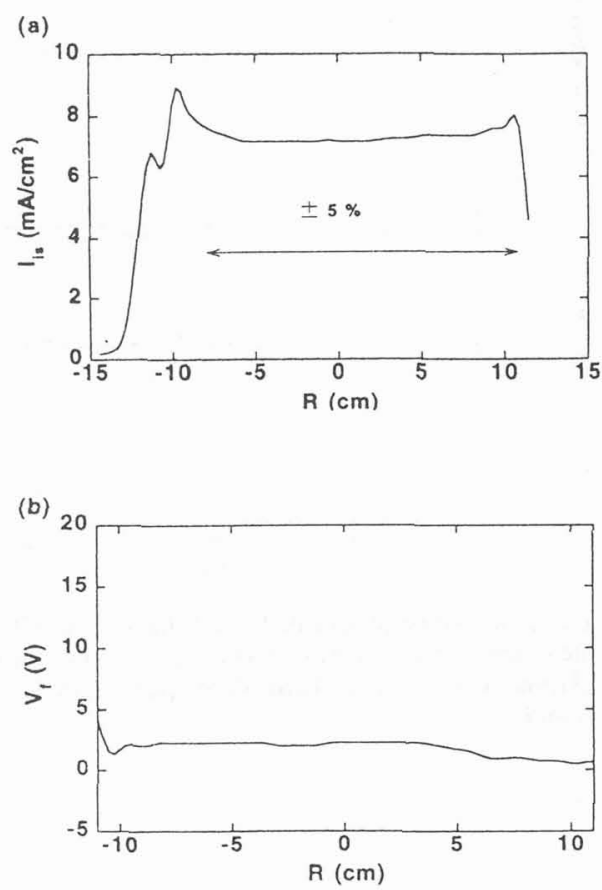

Fig. 2. Radial profile of (a) the ion saturation current density and (b) the floating potential for the uniform plasma. The input microwave power and gas pressure were $470 \mathrm{~W}$ and $2 \mathrm{~mm}$ Torr, respectively. 
(a)

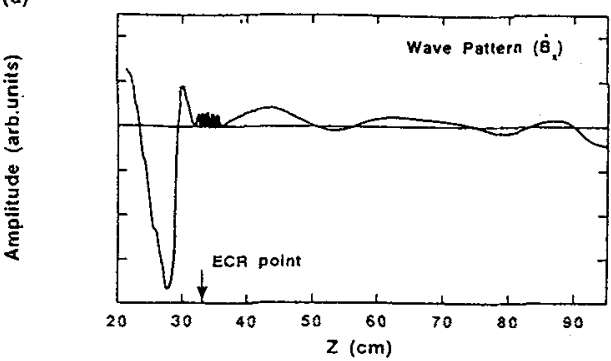

(b)

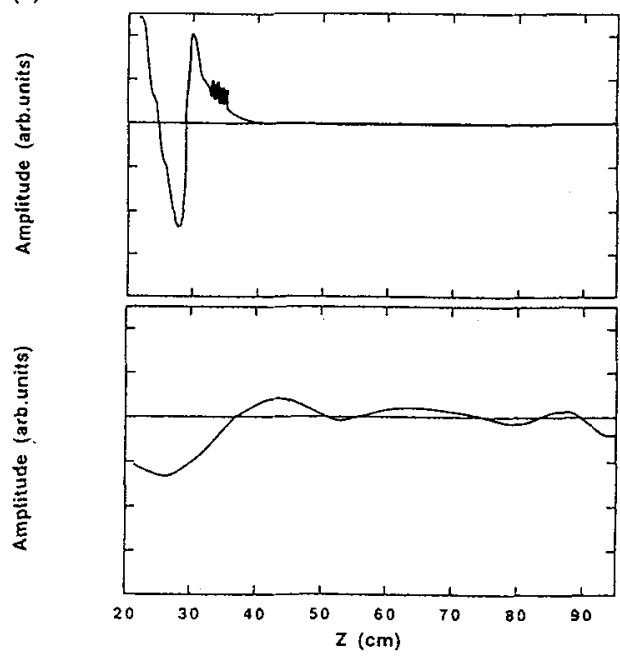

Fig. 3. (a) Wave pattern of $B_{x}$ and the axial profile of the output of the power meter in the case of the uniform plasma. (b) Wave patterns decomposed graphically from $B_{x}$. (a)

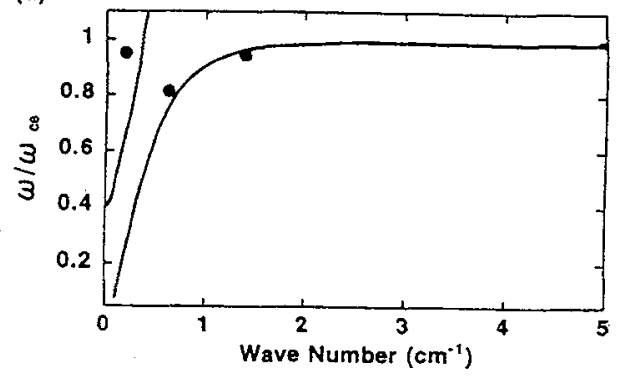

(b)

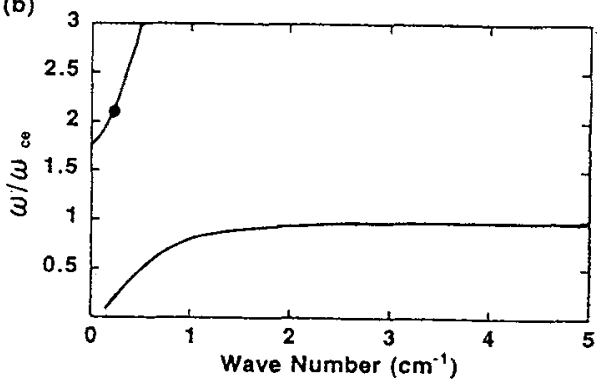

Fig. 4. Dispersion curves (a) before the ECR point and (b) after the ECR point, where solid lines are the theoretical dispersion curves.

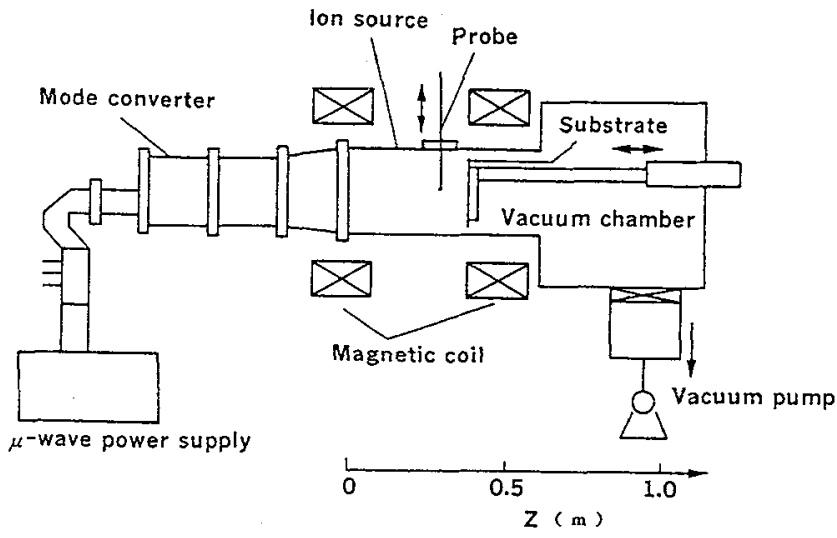

Fig. 5. Schematic diagram of the experimental apparatus. 


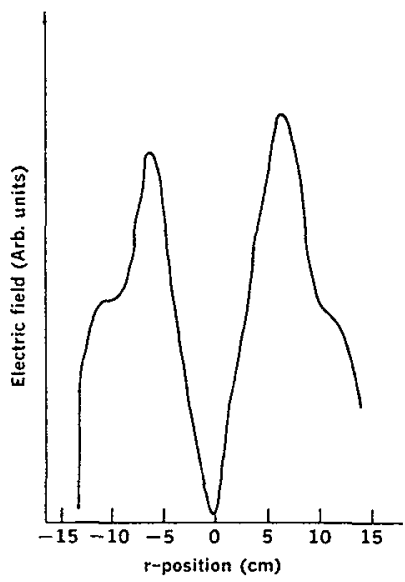

(a)

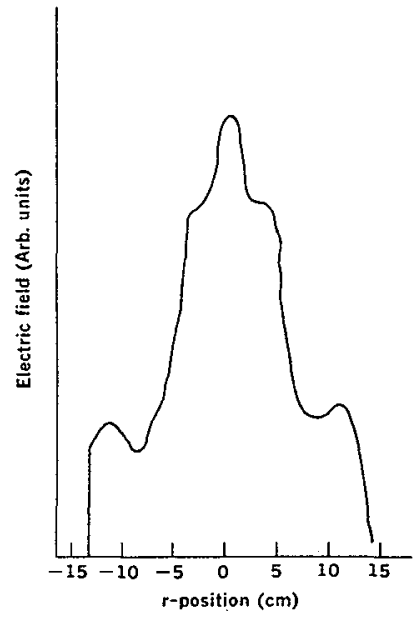

(b)

Fig. 6. Measured electric field distributions of the microwave emitted from (a) $\mathrm{TE}_{01}$ and (b) $\mathrm{TE}_{11}$ mode.

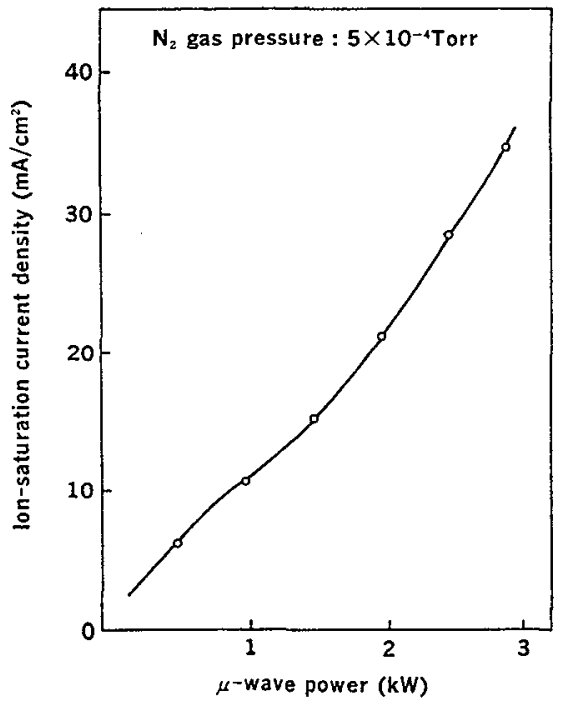

Fig. 7. Dependence of the ion saturation current density on the input microwave power, where the nitrogen gas pressure is $5 \times 10^{-4}$ Torr. 


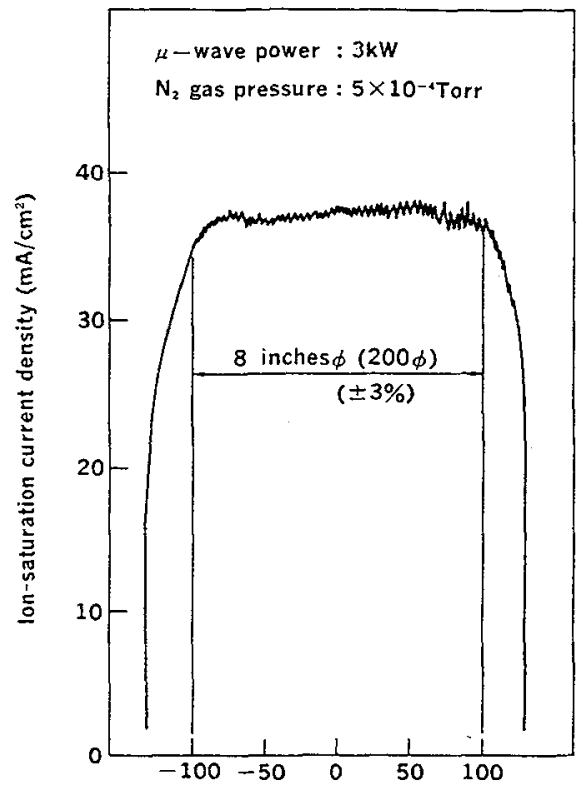

(a)

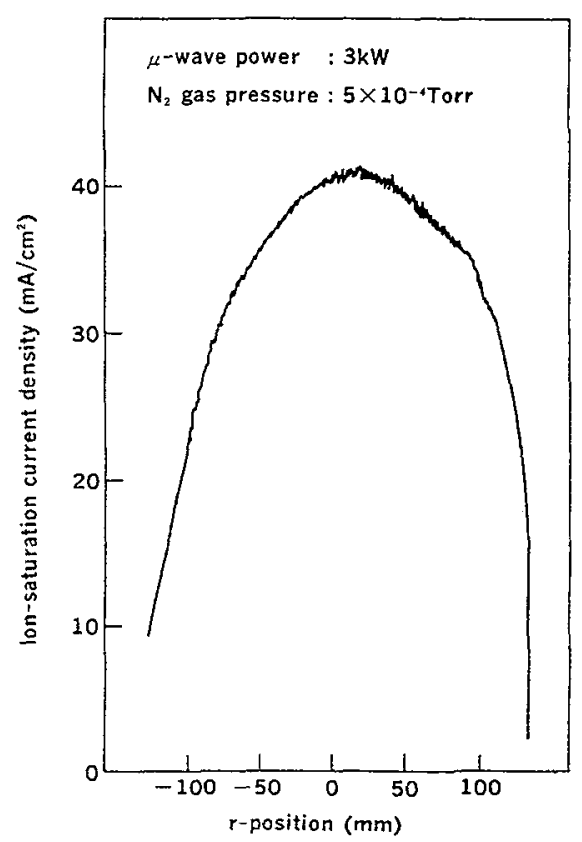

(b)

Fig. 8. Radial distributions of the ion saturation current density in the case of the (a) $\mathrm{TE}_{01}$ and (b) $T E_{11}$ mode, where the input microwave power is $3 \mathrm{~kW}$.

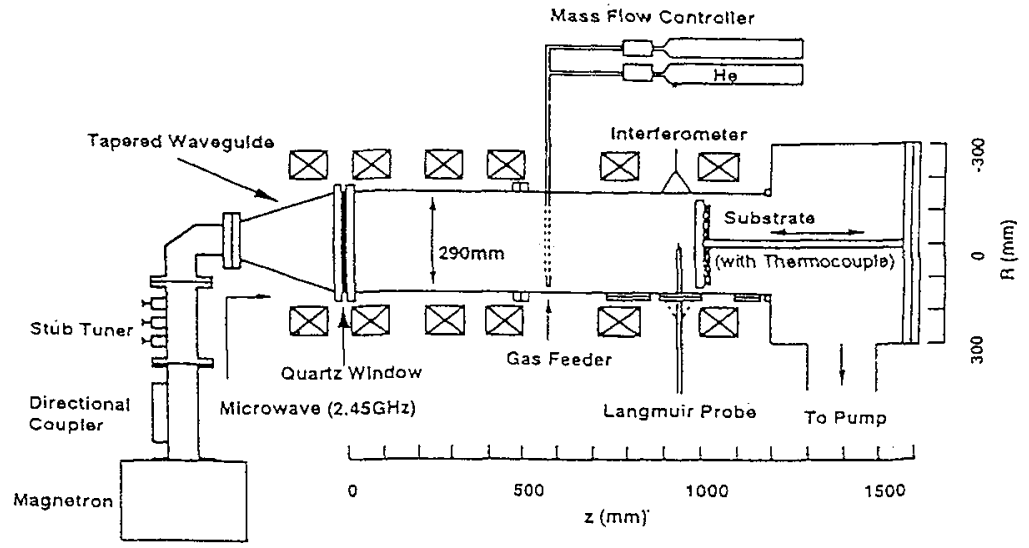

Fig. 9. Schematic diagram of the experimental apparatus. 


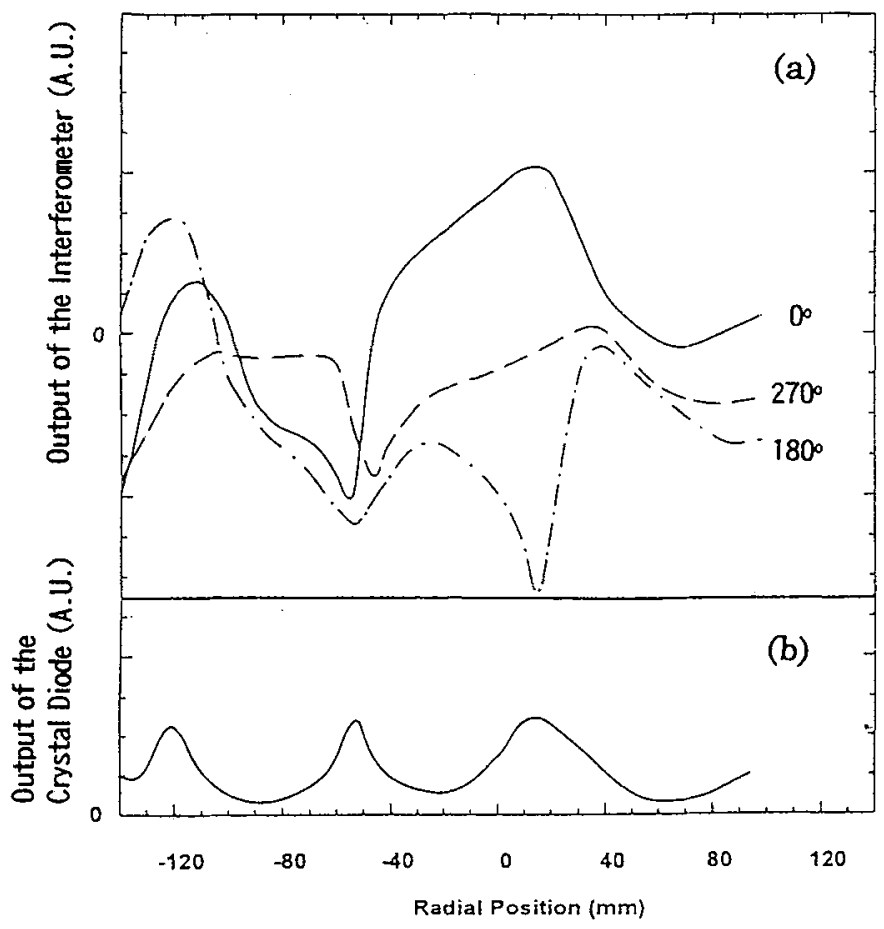

Fig. 10. (a) Radial wave patterns for different phases and (b) the output of the crystal diode, where the input microwave power is $0.8 \mathrm{~kW}$ and the pressure is $2 \mathrm{mTorr}$. The electron temperature is $5.3 \mathrm{eV}$.

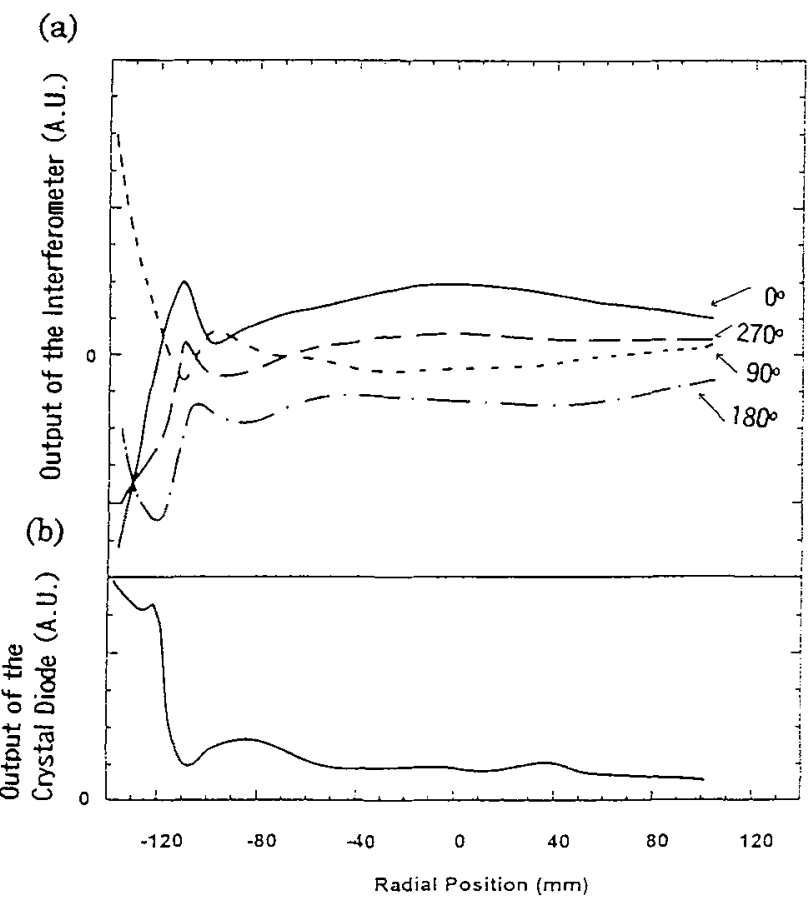

Fig. 11. (a) Radial wave patterns for different phases and (b) the output of the crystal diode, where the input microwave power is $1.5 \mathrm{~kW}$ and the pressure is $2 \mathrm{mTorr}$. The temperature and density of electrons are $8.5 \mathrm{eV}$ and $(1-2) \times 10^{17} \mathrm{~m}^{-3}$, respectively. 


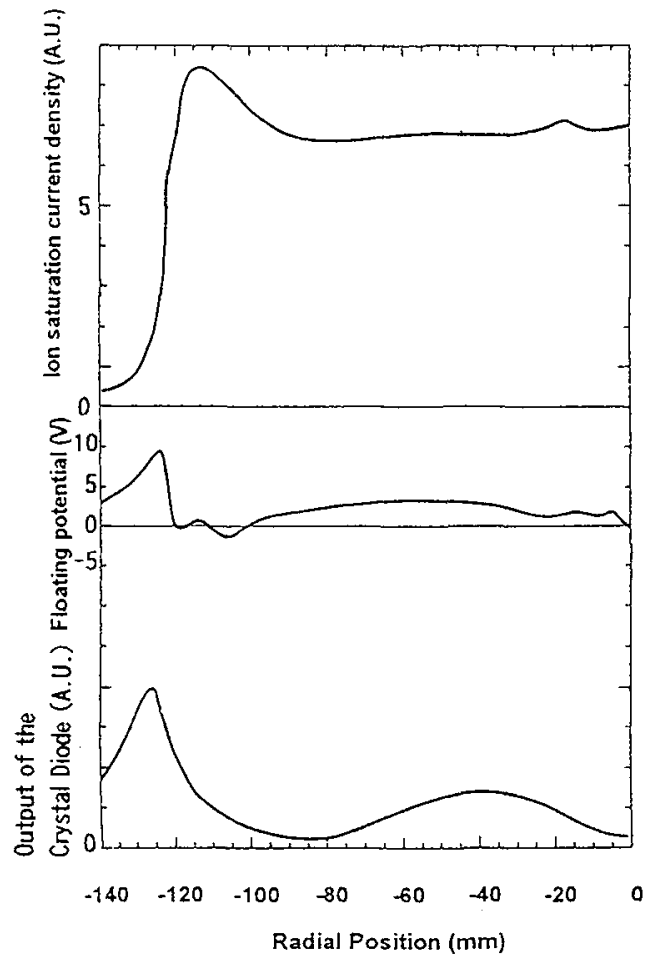

Fig. 12. The radial profiles of the ion saturation current density, floating potential, output of the crystal diode where the electric field is large near the chamber wall.

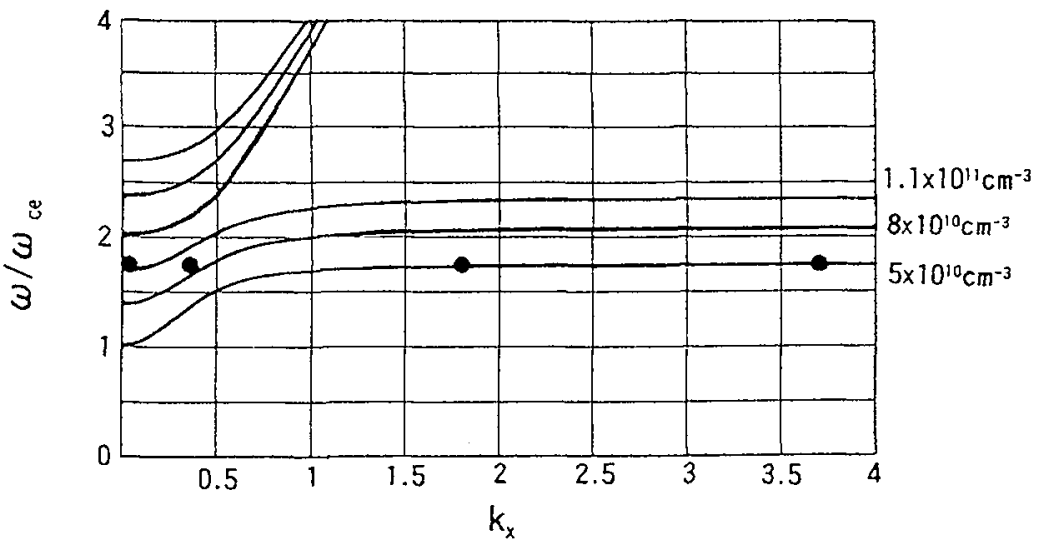

Fig. 13. The dispersion relations of the $\mathrm{X}$ wave for different electron densities. The closed circles and the solid lines correspond to the experimental values estimated from Fig. 10 and Fig. 11 and the theoretical dispersion relation of the $\mathrm{X}$ wave, respectively. 